\title{
To give or not to give? Blood for pediatric trauma patients prior to pediatric trauma center arrival
}

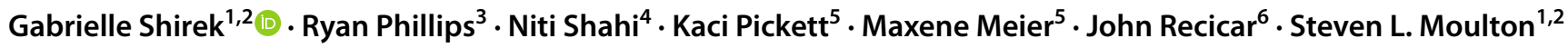

Accepted: 13 September 2021 / Published online: 4 October 2021

(c) The Author(s), under exclusive licence to Springer-Verlag GmbH Germany, part of Springer Nature 2021

\begin{abstract}
Purpose This study evaluates the indications, safety and clinical outcomes associated with the administration of blood products prior to arrival at a pediatric trauma center (prePTC).

Methods Children ( $\leq 18$ years) who were highest level activations at an ACS level 1 pediatric trauma center (PTC) from 2009-2019 were divided into groups:(1) patients with transport times $<4 \mathrm{~h}$ who received blood prePTC(preBlood) versus (2) age matched controls with transport times $<4 \mathrm{~h}$ who only received crystalloid prePTC (preCrystalloid).

Results Of 1269 trauma activations, 38 met preBlood and 38 met preCrystalloid inclusion criteria. A similar volume of prePTC crystalloid infusion was observed between cohorts $(p=0.311)$. PreBlood patients evidenced greater hemodynamic instability as demonstrated by higher prePTC pediatric age-adjusted shock index (SIPA) scores. PreBlood patients showed improvement in lactate $(p=0.038)$ and hemoglobin $(p=0.041)$ levels upon PTC arrival. PreBlood patients received less crystalloid within $12 \mathrm{~h}$ of PTC admission $(p=0.017)$. No significant differences were found in blood transfusion volumes within six $(p=0.293)$ and twenty-four $(p=0.575)$ hours of admission, nor in mortality between cohorts $(p=0.091)$.

Conclusions The administration of blood to pediatric trauma patients prior to arrival at a PTC is safe, transiently improves markers of shock, and was not associated with worse outcomes.
\end{abstract}

Keywords Crystalloid $\cdot$ Blood transfusion $\cdot$ Pediatric trauma $\cdot$ Prehospital

\section{Introduction}

Hemorrhage is associated with approximately $30 \%$ of traumatic deaths and is a leading cause of potentially preventable death $[1,2]$. Current American College of Surgeons (ACS) Advanced Trauma Life Support (ATLS) guidelines for the

Gabrielle Shirek

gabrielle.shirek@gmail.com

Ryan Phillips

ryanphillips4848@gmail.com

Niti Shahi

niti12@gmail.com

Kaci Pickett

kaci.pickett@cuanschutz.edu

Maxene Meier

maxene.meier@cuanschutz.edu

John Recicar

john.recicar@childrenscolorado.org

Steven L. Moulton

steven.moulton@childrenscolorado.org management of acute hemorrhage and hemorrhagic shock recommend an initial $20 \mathrm{~mL} / \mathrm{kg}$ bolus of an isotonic fluid followed by an early balanced resuscitation with blood products [3]. Despite these recommendations, many pediatric patients are resuscitated with far more than $20 \mathrm{~mL} / \mathrm{kg}$ of crystalloid before blood is given $[4,5]$. Trauma-induced coagulopathy

1 Division of Pediatric Surgery, Children's Hospital Colorado, Anschutz Medical Campus, 13213 E 16th Ave, Box 323, Aurora, CO 80045, USA

2 Department of Surgery, University of Colorado School of Medicine, Aurora, CO, USA

3 Department of Surgery, Ochsner Clinic Foundation, New Orleans, LS, USA

4 Department of Surgery, University of Massachusetts, Worcester, MA, USA

5 The Center for Research in Outcomes for Children's Surgery (ROCS), Center for Children's Surgery, University of Colorado School of Medicine, Aurora, CO, USA

6 Division of Nursing, Children's Hospital Colorado, Aurora, CO, USA 
may rapidly develop after severe injury and initiating crystalloid resuscitation without the inclusion of balanced blood product transfusion has been associated with worsening acidosis and ensuing suppression of essential coagulation factors $[6,7]$. As a result, crystalloid resuscitation strategies intended to treat hypovolemia may worsen acidosis, coagulopathy, and shock [7]. Prior work has also demonstrated that children may be more susceptible to trauma-induced coagulopathy $[6,7]$. By halving the volume of crystalloid and using an early, balanced resuscitation with blood products, physicians caring for traumatically injured adult patients have seen a reduction in acidosis and subsequent improvement in coagulation parameters [7].

Improved outcomes have been shown in adult trauma patients when restrictive prehospital crystalloid resuscitation is combined with a balanced, hemostatic resuscitation using blood products [8-10]. In the few pediatric studies investigating restrictive crystalloid use with early administration of blood products, overall improvement in several markers of coagulopathy and shock have been seen [11-13]. These studies by Moors et al. [11], Potter et al. [12], and Fahy et al. [13] highlight the safety of transfusing pediatric patients prior to arrival at a pediatric trauma center (prePTC), with no transfusion reactions reported. Despite these positive findings, prePTC blood transfusion protocols for pediatric trauma patients have not gained widespread adoption. This is partly due to the scarcity of data surrounding the indications, safety, outcomes, and mortality associated with prehospital transfusion in the pediatric population [13]. Another hinderance may be the perceived complexity of maintaining and safely transfusing blood products in emergency, mobile environments.

The current study aims to evaluate the indications, safety, and clinical outcomes associated with prePTC blood transfusion in pediatric trauma patients. We chose to compare patients who received blood products prior to arrival at a PTC (preBlood), to a cohort of patients who received crystalloid alone (preCrystalloid) — but who ultimately received blood within $6 \mathrm{~h}$ of PTC arrival. By comparing the first group to the latter, we sought to create a comparison cohort with similar illness severity that might have benefited from earlier transfusion in the prePTC setting. We hypothesized that pediatric patients who received prePTC blood products would have improved shock indices and less coagulopathy on arrival at the PTC, decreased in-hospital transfusion requirements, improved clinical outcomes, and lower mortality, compared to those who received crystalloid alone prior to arrival at the PTC.

\section{Material and methods}

\section{Setting}

Children's Hospital Colorado (CHCO) is the only American College of Surgeons verified Level 1 PTC in Colorado and serves the neighboring states of Montana, Wyoming, North and South Dakota, Nebraska, and New Mexico. The Colorado Multiple Institutional Review Board approved this study under a waiver of informed consent.

\section{Study design and population}

A retrospective review was conducted of all highestlevel trauma activations at CHCO between January 2009 to December 2019 to identify patients ( $\leq 18$ years), who arrived at the PTC within $4 \mathrm{~h}$ from the time of their injury. Patients were included if brought directly from the scene or as a transfer from an outside hospital. The timeframe of $4 \mathrm{~h}$ from the time of injury to arrival at the PTC was chosen because this is the approximate maximum transport time when patients are transported directly from the scene to our trauma center. Patients were placed into one of two groups based on the resuscitative fluids they were given in the prePTC setting. The preBlood group received crystalloid and blood products prior to arrival at the PTC, whereas the preCrystalloid group only received crystalloid. The preCrystalloid patient cohort was further narrowed to those receiving a blood product transfusion within $6 \mathrm{~h}$ of arrival to the PTC. This additional selection criterion produced a comparison cohort that required transfusion soon after their arrival at the PTC. Patients were excluded if they had missing transport times, missing transfusion volume or crystalloid volume data, and if $>18$ years old. The matched cohort was created using a nearest neighbor statistical matching algorithm with a Euclidean measure of distance to match patients by transport time (defined as the time from injury to arrival at the PTC.)

\section{Data collection}

Patient data were collected through an electronic medical record bioinformatics inquiry and an additional review of patient electronic medical records. Data included demographics, prePTC vital signs, Glasgow Coma Scale (GCS), time from injury to PTC arrival, PTC arrival vital signs, injury type, referring facility lab values, PTC admission lab values, and injury severity score (ISS). Additionally, total crystalloid volumes infused before arrival to the PTC and within the first $12 \mathrm{~h}$ following admission to the PTC were collected. Crystalloid administration volumes were further classified as low (0-39.9 mL/kg), mid (40-59.9 mL/kg), and high $(\geq 60 \mathrm{~mL} / \mathrm{kg})$, as previously defined by the literature [4]. Blood volumes and product types [red blood cells (RBC), fresh frozen plasma (FFP), and platelets (PLT)] were also recorded. Complications and clinical outcomes were documented.

Patient heart rate (HR) and systolic blood pressure (SBP) measurements were used to calculate SIPA scores in the field 
and upon PTC arrival. Shock index pediatric age-adjusted (SIPA) is a well-validated measure for identifying shock in pediatric trauma patients and has been shown to be superior to traditional, age-adjusted vital signs [14]. Elevated prehospital SIPA scores herald poor patient outcomes, as does trending prehospital SIPA scores over time [15]. Elevated in-hospital SIPA scores are associated with the need for transfusion, worse patient outcomes and increased mortality [16]. SIPA scores were compared to established cutoff values to categorically define the values as either elevated or not elevated [17, 18]. SIPA has only been validated in patients greater than 1 year of age. Therefore, normal ageappropriate HR and SBP values were used to calculate (HR/ SBP) cutoff values for patients less than 1 year-old.

\section{Statistical analysis}

Demographics, blood product and crystalloid volumes, and clinical outcomes data are presented as medians with interquartile ranges for continuous variables. Categorical variables are described as frequencies and proportions. Percent change was calculated for SIPA scores and lab values. Kruskal-Wallis or Chi-Square testing was done to evaluate group differences depending on the variable type. Statistical significance was defined as $p \leq 0.05$, with the $\alpha$ error level designated at 0.05 . All analyses were performed using R (R Foundation for Statistical Computing, Vienna, Austria, http://www.R-project.org/).

\section{Results}

\section{Demographics and injury characteristics}

During the study period, 1,269 patients arrived at the PTC as highest-level trauma activations. Thirty-eight patients who arrived at the PTC within $4 \mathrm{~h}$ from the time of their injury received a blood transfusion before arrival to the PTC; these patients comprised the preBlood cohort. Thirty-eight additional patients were matched to comprise the preCrystalloid group. All (38/38) preBlood patients and 79\% (30/38) of the preCrystalloid patients arrived as transfers to the PTC from an outside hospital. The median age was 7.5 years (IQR 2.1, 11.5 ) for the preBlood cohort and 5.5 years (IQR 2.5, 10.0) for the preCrystalloid patients. There was no significant difference in age between groups $(p=0.799)$. No significant differences were observed in gender, ISS, GCS, or trauma type (Table 1).

\section{Clinical characteristics}

Nearly all preBlood patients $(36 / 38,95 \%)$ had elevated SIPA scores immediately following injury, compared to $76 \%$ (29/38) of preCrystalloid patients $(p=0.050)$. There was no significant difference in the overall number of patients with elevated SIPA scores upon PTC arrival among the preBlood and preCrystalloid patients $(28 / 38,74 \%$ vs. $23 / 38,61 \%$,
Table 1 Demographics, injury, and clinical characteristics ${ }^{\mathrm{a}}$

\begin{tabular}{llll}
\hline & $\begin{array}{l}\text { PreBlood } \\
(n=38)\end{array}$ & $\begin{array}{l}\text { PreCrystalloid } \\
(n=38)\end{array}$ & $p$ value \\
\hline Age & $7.50(2.10,11.50)$ & $5.50(2.46,10.00)$ & 0.799 \\
Male Sex & $23(60.5 \%)$ & $21(55.3 \%)$ & 0.816 \\
Weight & $24.50(14.25,44.75)$ & $19.00(13.50,35.50)$ & 0.371 \\
ISS & $29.50(21.00,42.75)$ & $28.000(18.75,38.00)$ & 0.489 \\
GCS on arrival to PTC & $3.000(3.00,6.75)$ & $3.000(3.00,6.00)$ & 0.732 \\
TBI & $24(64.9 \%)$ & $25(65.8 \%)$ & 1.000 \\
NAT & $4(10.5 \%)$ & $8(21.1 \%)$ & 0.345 \\
GSW & $6(15.8 \%)$ & $2(5.3 \%)$ & 0.262 \\
BLSI & $7(18.4 \%)$ & $7(18.4 \%)$ & 1.000 \\
Time from injury to arrival at PTC & $2.87(2.08,3.58)$ & $2.742(2.10,3.13)$ & 0.352 \\
PTA SIPA & $1.91(1.45,2.68)$ & $1.42(1.29,1.94)$ & 0.006 \\
PTA SIPA elevated & $36(94.7 \%)$ & $29(76.3 \%)$ & 0.050 \\
ED SIPA & $1.39(1.08,1.92)$ & $1.29(0.98,1.62)$ & 0.226 \\
ED SIPA elevated & $28(73.7 \%)$ & $23(60.5 \%)$ & 0.329 \\
\hline
\end{tabular}

ISS Injury Severity Score; TBI Traumatic Brain Injury; NAT non accidental trauma; GSW gunshot wound; $B L S I$ blunt liver or spleen injury; PTA prior to arrival; SIPA shock index pediatric age adjusted; GCS Glasgow Coma Scale; FAST focused assessment with sonography for trauma; $E D$ emergency department; $C P R$ cardiopulmonary resuscitation; $P T C$ pediatric trauma center

${ }^{\mathrm{a}}$ Data are presented as medians with interquartile ranges Q1 \& Q3 for continuous variables. Categorical variables were described as frequencies and proportions. Kruskal-Wallis or Chi-Square testing was done depending on the variable type to determine $p$ values 
$p=0.329)$. The percent decrease in SIPA values from time of injury to arrival at the PTC was - 19\% (IQR - 45\%, 5\%) for the preBlood patients, versus - $11 \%$ (IQR - 28\%, 10\%) for the preCrystalloid patients.

\section{Blood and crystalloid volumes prior to arrival at the PTC}

The volume of crystalloid that the preBlood and preCrystalloid patients received prior to arrival at the PTC did not significantly differ [36.2 mL/kg $(25.0,51.5) \mathrm{vs} .29 .2 \mathrm{~mL} / \mathrm{kg}$ $(8.4,49.2), p=0.311]$. Similarly, prePTC crystalloid administration categories did not significantly differ between the groups, with $60 \%$ (23/38) of preBlood and 66\% (25/38) of preCrystalloid patients having received low-volume crystalloid administration. Eighteen percent (7/38) of preBlood and $13 \%(5 / 38)$ of preCrystalloid patients received mid-volume crystalloid administration, and $21 \%$ (8/38) of preBlood, and $24 \%$ (9/38) of preCrystalloid patients received high levels of crystalloid administration as defined in the methods. The median amount of blood product transfused in the preBlood patients prior to PTC arrival was $16.0 \mathrm{~mL} / \mathrm{kg}$ (IQR: 9.4 , 25.0). The breakdown of blood products transfused in the preBlood patients prior to arrival at the PTC was as follows:
$87 \%$ received pRBCs, $34 \%$ FFP, and $13 \%$ PLTs. Additional fluid data are shown in Table 2.

\section{Lab results}

PreBlood patients had significantly higher lactate levels [7.0 (IQR: 6.0, 10.0) vs. 3.5 (IQR: 3.3, 4.8) $p=0.011$ ] at the referring facilities, when compared to preCrystalloid patients. Upon arrival at the PTC there was no significant difference in initial lactate values between the two cohorts [2.9 (IQR: $1.8,4.3$ ) vs. 3.0 (IQR: 2.0, 4.9), $p=0.738$ ]. However, preBlood patients demonstrated a significant improvement in lactate following transport and arrival at the PTC [-62\% (IQR: $-75 \%,-48 \%$ ) when compared to the preCrystalloid cohort [-8\% (IQR: $-27 \%, 10 \%) ; p=0.038]$. Although there was no significant difference between the groups' base deficit (BD) values prior to PTC arrival, upon arrival at the PTC the preBlood patients had significantly lower BD values [- 9.5 (IQR: $-14.0,-6.9)$ vs. -6.0 (IQR: $-10.2,-4.7), p=0.004]$. Despite these findings, there was no significant difference in percent change for either group's $\mathrm{BD}$ values from prePTC to arrival at the PTC (preBlood: median 0\% (IQR: $-63 \%, 25 \%$ ); preCrystalloid: median $-22 \%$ (IQR: $-29 \%,-7 \%) ; p=0.892$ ).

Table 2 Blood product and transfusion data ${ }^{a}$

\begin{tabular}{|c|c|c|c|}
\hline & PreBlood $(n=38)$ & PreCrystalloid $(n=38)$ & $p$ value \\
\hline PTA crystalloid (mL/kg) & $36.165(25.00,51.50)$ & $29.17(8.41,49.17)$ & 0.311 \\
\hline PTA Blood (mL/kg) & $16.01(9.41,25.00)$ & NA & NA \\
\hline Received pRBC PTA & $33(86.8 \%)$ & NA & NA \\
\hline PTA pRBC (mL/kg) & $10.00(6.25,22.64)$ & NA & NA \\
\hline Received FFP PTA & $13(34.2 \%)$ & NA & NA \\
\hline PTA FFP $(\mathrm{mL} / \mathrm{kg})$ & $0.00(0.00,6.40)$ & NA & NA \\
\hline Received PLT PTA & $5(13.2 \%)$ & NA & NA \\
\hline PTA PLT (mL/kg) & $0.00(0.00,0.00)$ & NA & NA \\
\hline Total transufsion within $4 \mathrm{~h}$ of arrival at PTC $(\mathrm{mL} / \mathrm{kg})$ & $14.58(0.00,45.16)$ & $22.50(9.69,41.67)$ & 0.446 \\
\hline $\mathrm{pRBC}$ transfused within $4 \mathrm{~h}$ of arrival at PTC $(\mathrm{mL} / \mathrm{kg})$ & $11.19(0.00,30.33)$ & $15.81(7.43,33.65)$ & 0.336 \\
\hline FFP transfused within $4 \mathrm{~h}$ of arrival at PTC $(\mathrm{mL} / \mathrm{kg})$ & $0.00(0.00,17.04)$ & $0.00(0.00,17.56)$ & 0.777 \\
\hline PLT transfused within $4 \mathrm{~h}$ of arrival at PTC $(\mathrm{mL} / \mathrm{kg})$ & $0.00(0.00,0.00)$ & $0.00(0.00,0.00)$ & 0.646 \\
\hline Total transfusion within $6 \mathrm{~h}$ of arrival at PTC $(\mathrm{mL} / \mathrm{kg})$ & $17.71(1.99,55.52)$ & $26.97(13.49,41.67)$ & 0.293 \\
\hline $\mathrm{pRBC}$ transfused within $6 \mathrm{~h}$ of arrival at PTC $(\mathrm{mL} / \mathrm{kg})$ & $12.50(0.00,32.38)$ & $18.26(10.18,33.65)$ & 0.239 \\
\hline FFP transfused within $6 \mathrm{~h}$ of arrival at PTC (mL/kg) & $0.00(0.00,17.04)$ & $0.00(0.00,17.56)$ & 0.969 \\
\hline PLT transfused within $6 \mathrm{~h}$ of arrival at PTC $(\mathrm{mL} / \mathrm{kg})$ & $0.00(0.00,3.30)$ & $0.00(0.00,0.00)$ & 0.696 \\
\hline Total Transfusion within $24 \mathrm{~h}$ of arrival at PTC $(\mathrm{mL} / \mathrm{kg})$ & $28.21(8.69,55.52)$ & $27.92(15.16,41.67)$ & 0.575 \\
\hline $\mathrm{pRBC}$ transfused within $24 \mathrm{~h}$ of arrival at PTC $(\mathrm{mL} / \mathrm{kg})$ & $16.15(8.46,32.38)$ & $18.90(12.10,33.65)$ & 0.509 \\
\hline FFP transfused within $24 \mathrm{~h}$ of arrival at PTC $(\mathrm{mL} / \mathrm{kg})$ & $4.34(0.00,19.41)$ & $4.67(0.00,17.56)$ & 0.692 \\
\hline PLT transfused within $24 \mathrm{~h}$ of arrival at PTC $(\mathrm{mL} / \mathrm{kg})$ & $0.00(0.00,6.09)$ & $0.00(0.00,0.00)$ & 0.578 \\
\hline Crystalloid infused within $12 \mathrm{~h}$ of arrival at PTC & $52.25(30.86,96.71)$ & $86.82(52.15,123.04)$ & 0.017 \\
\hline
\end{tabular}

PTA prior to arrival; $P T C$ pediatric trauma center; $p R B C$ packed red blood cells; FFP fresh frozen plasma; $P L T$ platelet

${ }^{a}$ Data are presented as medians with interquartile ranges Q1 \& Q3 for continuous variables. Categorical variables were described as frequencies and proportions. Kruskal-Wallis or Chi-Square testing was done depending on the variable type to determine $p$ values 
PreBlood patients had significantly higher INR values at the referring facility, compared to preCrystalloid patients [1.5 (IQR: $1.5,1.8$ vs. 1.3 (IQR: $1.2,1.4$ ), $p=0.020$ ]; however, there was no significant difference at the PTC, where preBlood patient INR values had increased by $7 \%$ (IQR: $-1 \%, 16 \%$ ) and preCrystalloid INR values had increased by $10 \%$ (IQR: $9 \%, 19 \%$ ).

There was no significant difference in platelet counts or percent change in platelet counts between the two groups prior to arrival to the PTC. Upon arrival at the PTC, preBlood patients had significantly lower platelet counts compared to preCrystalloid patients [163.0 (IQR: 114.3, 200.8 ) vs. 269.0 (IQR: 169.0, 342.0), $p<0.001$ ], as shown in Table 3.

\section{Crystalloid volumes infused after arrival at the PTC}

Once at the PTC, preBlood patients received significantly less crystalloid $(52.3 \mathrm{~mL} / \mathrm{kg}$; IQR: $30.9,96.7)$ within $12 \mathrm{~h}$ of arrival compared to the preCrystalloid group $(86.8 \mathrm{~mL} / \mathrm{kg}$; IQR: 52.2, 123.0; $p=0.017)$. Similarly, within $12 \mathrm{~h}$ of arrival at the PTC, significantly more preBlood patients received low-level volumes (0-39.9 mL/kg) of crystalloid compared to preCrystalloid patients [14/38 (40\%) vs. 4/38 (11\%), $p=0.014]$. No significant difference existed in rates of mid-level crystalloid administration volumes $(40-59.9 \mathrm{~mL} /$ $\mathrm{kg}$ ) between preBlood and preCrystalloid patients [6/38 $(16 \%)$ vs. $7 / 38(18 \%), p=1.00]$. Notably, almost half of the preBlood patients $(17 / 38,45 \%)$ received high-volume crystalloid administration ( $\geq 60 \mathrm{~mL} / \mathrm{kg}$ ), which was significantly less than the preCrystalloid patients $(27 / 38,71 \%)$ $(p=0.036)$.

\section{Blood product transfusion after arrival at the PTC}

There were no significant differences between the two groups in the volumes of blood products transfused within the first six and $24 \mathrm{~h}$ of arrival at the PTC. Within the first $6 \mathrm{~h}$ of arrival, preBlood and preCrystalloid patients were transfused $17.7 \mathrm{~mL} / \mathrm{kg}$ and $27.0 \mathrm{~mL} / \mathrm{kg}$ of blood products, of which pRBC accounted for $12.5 \mathrm{~mL} / \mathrm{kg}$ and $18.3 \mathrm{~mL} /$ $\mathrm{kg}$, respectively. Within the first $24 \mathrm{~h}$ of arrival at the PTC the preBlood and preCrystalloid patients were transfused $28.2 \mathrm{~mL} / \mathrm{kg}$ and $27.9 \mathrm{~mL} / \mathrm{kg}$ of blood products, respectively, of which $16.2 \mathrm{~mL} / \mathrm{kg}$ and $18.9 \mathrm{~mL} / \mathrm{kg}$ were pRBC, and $4.3 \mathrm{~mL} / \mathrm{kg}$ and $4.2 \mathrm{~mL} / \mathrm{kg}$ were FFP. Further breakdown of blood product and crystalloid infusion volumes is shown in Table 2.

\section{Operation after arrival at the PTC}

Thirteen percent (5/38) of preBlood patients had an operation within $6 \mathrm{~h}$ of arrival at the PTC compared to $29 \%$
$(11 / 38)$ of preCrystalloid patients $(p=0.16)$. Sixteen percent (6/38) of preBlood patients and 32\% (12/38) of preCrystalloid patients went to the operating room within $12 \mathrm{~h}$ of arrival at the PTC $(p=0.18)$. Sixteen percent $(6 / 38)$ of preBlood and $37 \%(14 / 38)$ of preCrystalloid patients had an operation within $24 \mathrm{~h}$ of arrival at the PTC $(p=0.07)$. Laparotomies were performed in three $(8 \%)$ preBlood and one $(3 \%)$ preCrystalloid patients. Three $(8 \%)$ preBlood and nine (24\%) preCrystalloid patients had a craniectomy or craniotomy. Three preCrystalloid patients received the remaining procedures with one patient (3\%) undergoing an escharatomy, one patient (3\%) underwent femoral stabilization, and one patient (3\%) was taken to the operating room for incision and drainage.

\section{Complications and outcomes}

There were no transfusion reactions documented for preBlood patients in the prePTC setting. There was, however, one transfusion reaction in one preBlood patient and one in a preCrystalloid patient after arrival at the PTC. There were no significant differences in outcomes between the two groups, including pneumonia, acute renal failure, deep vein thrombosis, sepsis, ventilator days, intensive care unit (ICU) length of stay (LOS), hospital LOS, or mortality (Table 4).

\section{Discussion}

The administration of prePTC blood to pediatric trauma patients is safe. Patients who received prePTC blood did not experience a greater risk of complications (transfusion reaction, pneumonia, acute renal failure, deep vein thrombosis, sepsis), nor did they experience worse clinical outcomes (ventilator days, ICU LOS, hospital LOS, or mortality). These findings endorse prePTC transfusion safety and support a growing body of literature suggesting that harm can result from crystalloid-only resuscitation and delays to transfusion $[4,5,10,17]$. Interestingly, in comparison with the preBlood group, the preCrystalloid cohort received more blood and crystalloid within 6 and $12 \mathrm{~h}$ of arrival at the PTC, suggesting they might have benefited from earlier transfusion. This finding is bolstered by the observation that preBlood patients exhibited greater improvements in markers of shock and coagulopathy on arrival at the PTC. This is an important finding, considering hemorrhagic shock is a leading cause of preventable death in severely injured pediatric patients, who are at risk of trauma-induced coagulopathy $[1,6,7]$.

A study in the Netherlands evaluating the effectiveness of pRBC transfusion in the prehospital setting found that in a cohort of ten critically ill children, two of whom were 
Table 3 Lab results ${ }^{\mathrm{a}}$

\begin{tabular}{|c|c|c|c|}
\hline & PreBlood $(n=38)$ & PreCrystalloid $(n=38)$ & $p$ value \\
\hline PrePTC lactate & & & 0.011 \\
\hline $\mathrm{N}$-missing & 27 & 29 & \\
\hline Median(Q1,Q3) & $7.00(6.00,9.95)$ & $3.50(3.30,4.74)$ & \\
\hline ED lactate & & & 0.738 \\
\hline $\mathrm{N}$-missing & 20 & 19 & \\
\hline Median(Q1,Q3) & $2.91(1.83,4.32)$ & $3.00(1.99,4.87)$ & \\
\hline PrePTC base deficit & & & 0.360 \\
\hline $\mathrm{N}$-missing & 31 & 27 & \\
\hline Median(Q1,Q3) & $-8.00(-17.20,-7.00)$ & $-7.00(-8.70,-6.50)$ & \\
\hline ED base deficit & & & 0.004 \\
\hline N-missing & 0 & 1 & \\
\hline Median(Q1,Q3) & $-9.50(-14.00,-6.85)$ & $-6.00(-10.20,-4.70)$ & \\
\hline PrePTC INR & & & 0.020 \\
\hline $\mathrm{N}$-missing & 25 & 33 & \\
\hline Median(Q1,Q3) & $1.50(1.47,1.79)$ & $1.34(1.23,1.40)$ & \\
\hline ED INR & & & 0.280 \\
\hline $\mathrm{N}$-missing & 7 & 3 & \\
\hline Median(Q1,Q3) & $1.57(1.36,1.83)$ & $1.49(1.34,1.69)$ & \\
\hline PrePTC platelet count & & & 0.263 \\
\hline $\mathrm{N}$-missing & 14 & 22 & \\
\hline Median(Q1,Q3) & $299.50(235.50,325.00)$ & $307.00(268.00,418.25)$ & \\
\hline ED platelet count & & & $<0.001$ \\
\hline $\mathrm{N}$-missing & 4 & 2 & \\
\hline Median(Q1,Q3) & $163.00(114.25,200.75)$ & $269.00(169.00,342.00)$ & \\
\hline PrePTC hemoglobin & & & 0.472 \\
\hline $\mathrm{N}$-missing & 11 & 20 & \\
\hline Median(Q1,Q3) & $11.10(8.55,12.90)$ & $11.55(10.20,13.00)$ & \\
\hline ED hemoglobin & & & 0.147 \\
\hline $\mathrm{N}$-missing & 1 & 1 & \\
\hline Median(Q1,Q3) & $11.20(8.80,14.00)$ & $9.60(8.20,11.60)$ & \\
\hline PrePTC PTT & & & 0.103 \\
\hline $\mathrm{N}$-missing & 28 & 34 & \\
\hline Median(Q1,Q3) & $45.80(33.38,56.73)$ & $29.00(28.18,30.58)$ & \\
\hline ED PTT & & & 0.088 \\
\hline $\mathrm{N}$-missing & 5 & 3 & \\
\hline Median(Q1,Q3) & $40.00(31.00,60.50)$ & $34.00(29.00,41.75)$ & \\
\hline ED fibrinogen & & & 0.259 \\
\hline $\mathrm{N}$-missing & 21 & 27 & \\
\hline Median(Q1,Q3) & $125.00(80.00,196.00)$ & $166.00(120.00,231.00)$ & \\
\hline ED D-DIMER & & & 0.970 \\
\hline $\mathrm{N}$-missing & 23 & 31 & \\
\hline Median(Q1,Q3) & $20.00(11.83,20.00)$ & $20.00(9.70,20.00)$ & \\
\hline ED white blood cell count & & & 0.058 \\
\hline $\mathrm{N}$-missing & 4 & 2 & \\
\hline Median(Q1,Q3) & $13.00(9.60,20.14)$ & $17.83(11.92,23.72)$ & \\
\hline
\end{tabular}

PrePTC Prior to arrival to the pediatric trauma center; ED emergency department; INR international normalized ratio; $P T T$ partial thromboplastin time

${ }^{a}$ Data are presented as medians with interquartile ranges Q1 \& Q3 for continuous variables. Categorical variables were described as frequencies and proportions. Kruskal-Wallis or Chi-Square testing was done depending on the variable type to determine $p$ values 
Table 4 Outcomes ${ }^{\mathrm{a}}$

\begin{tabular}{llll}
\hline & PreBlood $(n=38)$ & PreCrystalloid $(n=38)$ & $p$ value \\
\hline Pneumonia & $5(13.2 \%)$ & $4(10.5 \%)$ & 1.000 \\
Extremity Compartment Syn- & $0(0.0 \%)$ & $0(0.0 \%)$ & 1.000 \\
$\quad$ drome & $3(7.9 \%)$ & $2(5.3 \%)$ & 1.000 \\
Urinary tract infection & $1(2.6 \%)$ & $0(0.0 \%)$ & 1.000 \\
Acute renal failure & $0(0.0 \%)$ & $1(2.6 \%)$ & 1.000 \\
Deep vein thrombosis & $0(0.0 \%)$ & $1(2.6 \%)$ & 1.000 \\
Sepsis & $5.00(1.00,8.00)$ & $4.00(1.00,8.00)$ & 0.798 \\
Ventilator days & $6.00(2.00,13.00)$ & $5.50(3.00,10.75)$ & 0.694 \\
ICU LOS & $6.50(2.00,15.75)$ & $10.00(5.00,17.75)$ & 0.137 \\
Hospital LOS & $17(44.7 \%)$ & $9(23.7 \%)$ & 0.091 \\
Mortality & & &
\end{tabular}

$I C U$ intensive care unit; $L O S$ length of stay

${ }^{a}$ Data are presented as medians with interquartile ranges Q1 \& Q3 for continuous variables. Categorical variables were described as frequencies and proportions. Kruskal-Wallis or Chi-Square testing was done depending on the variable type to determine $p$ values

unexpected survivors, all had improved acid-base balance, hemoglobin levels, and coagulopathy [11]. In the current study, similar improved trends were found in markers of shock, including lactate and SIPA, as well as in hemoglobin levels and INR values. These results support prePTC blood safety and are consistent with extant studies that describe the potential for hemodilution and negation of blood product benefits that accompany high volume crystalloid administration [18].

Studies by Acker et al. [4] and Coons et al. [5] showed that an infused crystalloid volume of $>60 \mathrm{~mL} / \mathrm{kg}$ in the first $24 \mathrm{~h}$ was associated with worse outcomes, including increased hospital LOS, need for mechanical ventilation, severe anemia, and thrombocytopenia. Similarly, in a multicenter prospective observational study by Polites et al. [19] pediatric patients with hemorrhagic shock had increased odds of prolonged ventilator days, intensive care unit length of stay (LOS), and hospital LOS for each crystalloid bolus they were given before being transfused. Nearly a quarter of preBlood patients in the present study received a prePTC crystalloid resuscitation considered high-volume administration $(\geq 60 \mathrm{~mL} / \mathrm{kg}$ ), and nearly half received a high-volume crystalloid resuscitation within $12 \mathrm{~h}$ of PTC arrival. Conversely, preBlood patients received significantly less crystalloid than the preCrystalloid patients, which further emphasizes the potential benefits of prePTC blood transfusion. While no significant differences were found in patients who went to the operating room within 6,12 , and $24 \mathrm{~h}$ of arrival, descriptively more preCrystalloid patients had operations after arrival at the PTC. This may account for some of the increased crystalloid volumes received by preCrystalloid patients following their arrival at the PTC.

Aggressive crystalloid resuscitation without blood product transfusion can dilute coagulation factors and worsen trauma-induced coagulopathy in adult and pediatric trauma populations [5, 18, 19]. Bickell et al. [17] imputed aggressive prehospital fluid resuscitation in perpetuating hemorrhage due to blood pressure elevation with the concomitant dilution of hemoglobin and clotting factors. PreBlood patients, despite receiving similar prePTC crystalloid volumes, had lower lactate values than the preCrystalloid cohort. Additionally, while preBlood patients demonstrated no change in their base deficits, preCrystalloid patients had widening derangements in BD values. PreBlood patients also showed improvement in their hemoglobin levels upon arrival at the PTC, whereas preCrystalloid patients' hemoglobin levels dropped. The improvement seen in preBlood patients' SIPA scores on arrival at the PTC are indicative of an improvement in their shock status, most likely due to early blood product transfusion.

Downward trends in SIPA scores are associated with improved outcomes in pediatric patients suffering from blunt traumatic injuries [20]. In a study by Phillips et al. [21] traumatically injured pediatric patients with an elevated prehospital SIPA that remained elevated on arrival to the E.D. were more likely to receive a transfusion and more likely to have worse outcomes. The downward progression in SIPA values identified in the preBlood cohort suggests a benefit to giving blood early; however, we were unable to demonstrate improved outcomes in this group.

This study should inform future research in restrictive crystalloid resuscitation and increased use of prePTC blood for the pediatric population. These patients can be challenging to manage, especially in the prePTC setting. It is hard to determine which children will most benefit from early transfusion and hemostatic resuscitation, and even harder to predict when and which children will decompensate. This study therefore highlights the need for point-of-care testing and/or a noninvasive, real-time method for monitoring individual-specific hemodynamic status, to aid in the 
identification of children who will most benefit from early blood product administration.

\section{Limitations}

This study has several limitations. It is a retrospective study at a single center. The implementation of prePTC blood transfusion protocols is not widespread. Therefore, most patients were transferred from outside facilities. To best replicate transfer times from the injury scene, we limited our patient population to those with transport times under four hours. This significantly reduced the study sample size, as many of the patients transported to $\mathrm{CHCO}$ are referrals from neighboring states, with long scene, outside hospital and transport times. Additionally, some patients may have had their transfusion started at the referring hospital, whereas others during active transport. Oftentimes we were unable to establish the exact location and timing of transfusion initiation in external reports, further limiting our study population. Furthermore, nonsignificant differences $(p=0.05)$ were seen in the number of patients in each cohort with elevated SIPA scores, suggesting that patients in the preBlood group were sicker than patients in the preCrystalloid group, despite our best efforts to match the cohorts. Because of the small patient selection pool, patients were unable to be matched on multiple factors.

We were unable to determine in all cases which patients received coagulation factors and/or tranexamic acid prior to arrival at our facility. Neither were we able to evaluate the impact of different blood component ratios and their associations with clinical outcomes. Lab values measuring lactate, base deficit, coagulation factors, and platelet counts were unavailable for some patients. This is a significant limitation to our analysis of lab values and the changes that may have resulted following the administration of blood and/or crystalloid products in the prePTC setting. Despite this major limitation, the study still demonstrated the safety of earlier transfusion, which should promote future prospective investigation of this important topic.

Future studies should aim to include multiple centers and prospectively enroll patients from the scene of injury instead of including transfers from outside referral emergency departments, to better emulate conditions experienced by patients transported directly from the scene. Future studies should also aim to compare groups with transport times under one hour, which would be more representative of most pediatric trauma transports. Additionally, future research efforts should be directed towards randomized controlled trials to best evaluate the outcomes associated with prehospital blood administration and blood component products, with the implementation of standardized lab collection protocols with specific time intervals.

\section{Conclusion}

This study is the first to compare matched pediatric cohorts based on transport time, stratified by prePTC blood transfusion status. Importantly, it has provided evidence for the safety and efficacy of early blood transfusion. Specifically, traumatically injured pediatric patients who received prePTC blood transfusions were more likely to have improved shock markers and reduced crystalloid needs upon arrival at the PTC. Additionally, patients who received prePTC blood did not have an increased risk of worse clinical outcomes. Thus, this study adds to a growing body of evidence that restrictive crystalloid and early blood transfusion are mutually beneficial when managing and transporting severely injured children.

Author contributions Literature Search GS, RP, NS. Study conception and design GS, RP, NS, SM. Acquisition of data GS, RP, NS. Analysis and interpretation of data GS, RP, NS, KP, MM, JR, SM. Drafting of the manuscript GS. Critical revision of manuscript GS, RP, NS, KP, MM, JR, SM.

Funding No grant funding supported this research. Institutional funding was provided by the Children's Hospital Colorado, the Center for Research in Outcomes for Children's Surgery and the Colorado Firefighter Calendar.

\section{Declarations}

Conflict of interest The authors have no conflicts of interest to disclose.

Ethical approval All human studies have been approved by the appropriate ethics committee of the Colorado Multiple Institutional Review Board with a waiver of informed consent and have therefore been performed in accordance with the ethical standards laid down in the 1964 Declaration of Helsinki and its later amendments.

\section{References}

1. Trunkey D, Lim R (1974) Analysis of 425 consecutive trauma fatalities. J Am Coll Emerg Phys 69(3):368-371

2. Shackford SR, Mackersie RC, Holbrook TL, Davis JW, Hollingsworth-Fridlund P, Hoyt DB, Wolf PL (1993) The epidemiology of traumatic death. A population-based analysis. Arch Surg 128(5):571-575

3. Henry S. ATLS 10th edition offers new insights into managing trauma patients. June 1 2018. https://bulletin.facs.org/2018/06/ atls-10th-edition-offers-new-insights-into-managing-trauma-patie nts/\#Chapter_10_Pediatric_Trauma. Accessed June 10, 2020

4. Acker SN, Ross JT, Partrick DA, DeWitt P, Bensard DD (2014) Injured children are resistant to the adverse effects of early high volume crystalloid resuscitation. J Pediatr Surg 49(12):1852-1855

5. Coons BE, Tam S, Rubsam J, Stylianos S, Duron V (2018) High volume crystalloid resuscitation adversely affects pediatric trauma patients. J Pediatr Surg 53(11):2202-2208 
6. Hendrickson JE, Shaz BH, Pereira G, Atkins E, Johnson KK, Bao G, Easley KA, Josephson CD (2012) Coagulopathy is prevalent and associated with adverse outcomes in transfused pediatric trauma patients. J Pediatr 160(2):204-209.e3

7. Holcomb JB, Jenkins D, Rhee P, Johannigman J, Mahoney P, Mehta S, Cox ED, Gehrke MJ, Beilman GJ, Schreiber M et al (2007) Damage control resuscitation: directly addressing the early coagulopathy of trauma. J Trauma 62(2):307-310

8. Woolley T, Thompson P, Kirkman E, Reed R, Ausset S, Beckett A, Bjerkvig C, Cap AP, Coats T, Cohen M et al (2018) Trauma Hemostasis and Oxygenation Research Network position paper on the role of hypotensive resuscitation as part of remote damage control resuscitation. J Trauma Acute Care Surg 84(6S Suppl 1):S3-S13

9. Brown JB, Sperry JL, Fombona A, Billiar TR, Peitzman AB, Guyette FX (2015) Pre-trauma center red blood cell transfusion is associated with improved early outcomes in air medical trauma patients. J Am Coll Surg 220(5):797-808

10. Powell EK, Hinckley WR, Gottula A, Hart KW, Lindsell CJ, McMullan JT (2016) Shorter times to packed red blood cell transfusion are associated with decreased risk of death in traumatically injured patients. J Trauma Acute Care Surg 81(3):458-462

11. Moors XRJ, Bouman SJM, Peters JH, Smulders P, Alink MBO, Hartog DD, Stolker RJ (2018) Prehospital blood transfusions in pediatric patients by a helicopter emergency medical service. Air Med J 37(5):321-324

12 Potter DD, Berns KS, Elsbernd TA, Zietlow SP (2015) Prehospital use of blood and plasma in pediatric trauma patients. Air Med J 34(1):40-3

13. Fahy AS, Thiels CA, Polites SF, Parker M, Ishitani MB, Moir CR, Berns K, Stubbs JR, Jenkins DH, Zietlow SP, Zielinski MD (2017) Prehospital blood transfusions in pediatric trauma and nontrauma patients: a single-center review of safety and outcomes. Pediatr Surg Int 33(7):787-792

14. Acker SN, Bredbeck B, Partrick DA, Kulungowski AM, Barnett CC, Bensard DD (2017) Shock index, pediatric age-adjusted (SIPA) is more accurate than age-adjusted hypotension for trauma team activation. Surgery 161(3):803-807
15. Nordin A, Shi J, Wheeler K, Xiang H, Kenney B (2019) Ageadjusted shock index: from injury to arrival. J Pediatr Surg 54(5):984-988

16. Linnaus ME, Notrica DM, Langlais CS, St Peter SD, Leys CM, Ostlie DJ, Maxson RT, Ponsky T, Tuggle DW, Eubanks JW 3rd et al (2017) Prospective validation of the shock index pediatricadjusted (SIPA) in blunt liver and spleen trauma: an ATOMAC+ study. J Pediatr Surg 52(2):340-344

17. Bickell WH, Wall MJ Jr, Pepe PE, Martin RR, Ginger VF, Allen MK, Mattox KL (1994) Immediate versus delayed fluid resuscitation for hypotensive patients with penetrating torso injuries. $\mathrm{N}$ Engl J Med 331(17):1105-1109

18. Duchesne JC, Heaney J, Guidry C, McSwain N Jr, Meade P, Cohen M, Schreiber M, Inaba K, Skiada D, Demetriades D et al (2013) Diluting the benefits of hemostatic resuscitation: a multiinstitutional analysis. J Trauma Acute Care Surg 75(1):76-82

19. Polites SF, Moody S, Williams RF, Kayton ML, Alberto EC, Burd RS, Schroeppel TJ, Baerg JE, Munoz A, Rothstein WB et al (2020) Timing and volume of crystalloid and blood products in pediatric trauma: an Eastern Association for the Surgery of Trauma multicenter prospective observational study. J Trauma Acute Care Surg 89(1):36-42

20. Vandewalle RJ, Peceny JK, Dolejs SC, Raymond JL, Rouse TM (2018) Trends in pediatric adjusted shock index predict morbidity and mortality in children with severe blunt injuries. J Pediatr Surg 53(2):362-366

21. Phillips R, Acker S, Shahi N, Shirek G, Meier M, Goldsmith A, Recicar J, Moulton S, Bensard D (2020) The shock index, pediatric age-adjusted (SIPA) enhanced: prehospital and emergency department SIPA values forecast transfusion needs for blunt solid organ injured children. Surgery 168(4):690-694

Publisher's Note Springer Nature remains neutral with regard to jurisdictional claims in published maps and institutional affiliations. 